\title{
Sexuality in psychosis: dysfunction, risk and mental capacity
}

\author{
Shubulade Smith \& Daniel Herlihy
}

\begin{abstract}
SUMMARY
Issues such as sexual dysfunction, hypersexuality, risky sexual behaviour and mental capacity in relation to sexual activities arise frequently in people with psychosis. However, they may not always be given a priority in doctor-patient interactions, despite their often critical importance from the patient's perspective. Having a working knowledge of these topics may improve the quality of psychiatric intervention for people with severe mental illness. This article explores these topics, with a particular emphasis on assessment and management in everyday practice.
\end{abstract}

\section{DECLARATION OF INTEREST}

S.S. has received unrestricted educational grants from AstraZeneca and Eli Lilly, and speaker and consultancy fees from AstraZeneca, Bristol-Myers Squibb, Eli Lilly, Janssen and Sanofi-Synthélabo.

A satisfactory sex life requires an ability to socially interact with others, an understanding and acceptance of one's sexual orientation, a certain level of self-confidence and adequately functioning sexual physiology (Box 1). Psychoses (primarily schizophrenia and bipolar disorder) are characterised by disorders in basic perceptual, cognitive, affective and judgemental processes. Those affected experience delusions, hallucinations, disorganised speech and disorganised behaviour. It seems likely, therefore, that psychotic disorders will be associated with difficulties in sexual functioning, as they interfere with psychological and social interaction.

\section{Sexual dysfunction in psychosis}

Sexual dysfunction in psychosis involves a complex interplay of biological, psychological and social factors that may or may not relate to the underlying illness and its treatment. Some patients will require more specialist assessment and management. However, the initial assessment should be undertaken by the treating psychiatrist, who is well placed to manage sexual dysfunction when caused by the drug treatment of psychosis, or to refer for specialist advice if this is indicated.

\section{Sexual dysfunction as a side-effect of drug treatment of psychosis}

Despite the improvement in interpersonal functioning that can be produced by antipsychotic drugs, there is evidence that people receiving medication for schizophrenia frequently suffer sexual dysfunction (Aizenberg 1995; Smith 2002).

Gitlin (1994) lists four ways in which psychotropic medication can affect sexual function:

- direct effect on the central nervous system (CNS), e.g. the dopaminergic increase caused by levodopa, resulting in increased libido;

- indirect CNS effects, e.g. sedation secondary to histaminic effects of antipsychotics, resulting in decreased sexual activity;

- peripheral effects, e.g. $\alpha$-adrenergic blockade, resulting in priapism;

- hormonal effects, e.g. dopamine blockade causes hyperprolactinaemia, which has negative effects on sexual and reproductive function.

Reviewing whether the receptor-binding properties of the prescribed antipsychotic match the type of sexual dysfunction being experienced will assist in determining whether a patient's sexual dysfunction is a result of their prescribed treatment.

B0X 1 Normal sexual function

- Sexual function is divided into a number of different areas:

sexual interest or libido

arousal

plateau

orgasm/ejaculation

resolution

- In males, arousal is essentially the ability to achieve and maintain an erection

- In females, there is relaxation, swelling and lubrication of the vaginal walls in readiness for penetration

- The orgasm refers to the mental and physical phenomena that signify the climax of coital activity. In males, this is accompanied by the ejaculation of seminal fluid

\section{ARTICLE}

Shubulade Smith is a consultant psychiatrist and clinical senior lecturer at the Maudsley Hospital and Institute of Psychiatry, King's College London. Her research interests include endocrine and reproductive side-effects of antipsychotic medication, physical health in severe mental illness and the short-term management of violent behaviour. Daniel Herlihy recently completed his higher training in psychiatry at the South London and Maudsley NHS Foundation Trust, London. His research interests include functional outcomes in psychosis. Correspondence Dr Shubulade Smith, Department of Forensic and Neurodevelopmental Science, PO

Box 23, Institute of Psychiatry, De Crespigny Park, London SE5 8AF, UK. Email: s.smith@iop.kcl.ac.uk 


\section{Conventional (typical) antipsychotic medications}

Antipsychotics differ in their ability to block adrenergic, histaminic and muscarinic receptors, and therefore differ in their interference with sexual function. Sexual dysfunction is more likely to be seen with high-dose, low-potency drugs such as the aliphatic phenothiazine chlorpromazine. These tend to have less potent dopamine-blocking ability, but significant anti-adrenergic and anticholinergic activity (Kotin 1976; Lingjaerde 1987). Antimuscarinic activity promotes dryness of the mucous membranes, which may cause discomfort during coital activity. In men, the anti-adrenergic effects of antipsychotics include ejaculatory dysfunction and priapism (Mitchell 1983).

Raised prolactin levels are associated with sexual dysfunction in both women and men (Ghadirian 1982). However, a preliminary study found that women have a greater prolactin response to antipsychotics compared with men and therefore may be more likely to suffer sexual dysfunction associated with prolactin increase (Nathan 1983). This has been confirmed by subsequent research, which showed that rates of sexual dysfunction in antipsychotic-treated individuals were high and that, in women, dysfunction was particularly related to the prolactin-inducing effects of the medication (Smith 2002).

The well-established physiological and pathological sequelae of sustained high prolactin levels include hypogonadal states with decreased libido, erectile and ejaculatory dysfunction, decreased vaginal response, anorgasmia, menstrual irregularities, galactorrhoea and reduced fertility.

\section{Atypical antipsychotic medications}

The newer, second-generation antipsychotics (the atypicals) initially appeared to have less troublesome side-effect profiles than the older drugs. Dopamine (excitatory) and serotonin (inhibitory) are both important neurotransmitters in sexual function. The reciprocal activity of these neurotransmitters in the atypicals seems to reduce the likelihood of raised prolactin levels, and this may be associated with lesser sexual dysfunction. Atypical drugs differ in their effects on prolactin, and divide into those that raise prolactin levels and those that do not. Both olanzapine and quetiapine have been associated with low levels of reported sexual dysfunction (Beasley 1997; Keller 2002). It is of note that both drugs tend not to produce marked increases in prolactin, in common with clozapine, which, together with quetiapine and aripiprazole, is truly prolactin-sparing. Clozapine has been found to be associated with low levels of sexual dysfunction compared with typical antipsychotics, although there have been reports of priapism with this drug (Rosen 1992; Seftel 1992, Compton 2000).

Risperidone has repeatedly been shown to be associated with significant levels of sexual dysfunction, particularly ejaculatory disturbance (Kim 2002; Bobes 2003; Knegtering 2004). Sertindole is also associated with ejaculatory disturbance, mainly absent ejaculate (Lewis 2000). This is thought to be a result of $\alpha$-adrenergic blockade.

It is very likely that the endocrine sequelae of antipsychotic medication have been underestimated. Given the high rate of hyperprolactinaemia among patients taking antipsychotics, hypogonadal states are a likely consequence. In addition to causing sexual dysfunction, this may contribute to the reduced fertility rates seen in schizophrenia (Rao 1980; Smith 2003).

\section{Anticholinergic medication}

Very little is known about the effects of anticholinergic medication on sexual function. However, it is likely that concurrent prescription of anticholinergics with antipsychotics will potentiate the effects of antipsychotics on arousal, and may cause impotence or failure of the vaginal response.

\section{Mood stabilisers}

Lithium tends not to be associated with sexual dysfunction. One study found that some men taking lithium reported reduced sexual interest, but most who were taking lithium alone had no difficulties with their sexual function (Aizenberg 1996). However, when lithium is taken in combination with other medications, there is a greater risk of sexual dysfunction (Ghadirian 1992; Aizenberg 1996). The enzyme-inducing anti-epileptics that are used as mood stabilisers (sodium valproate, carbamazepine) are associated with sexual dysfunction, and this is thought to be a hormonal effect, via suppression of the hypothalamicpituitary-gonadal axis. Lamotrigine, however, appears to be far less likely to cause sexual dysfunction (Lambert 2001).

\section{Psychological and social factors implicated in sexual dysfunction}

The finding that poor premorbid sociosexual functioning is an important determinant of longterm outcome lends weight to the argument that lowered fertility rates in schizophrenia are a result of its social, rather than biological, effects (Keefe 1989; Jones 1993). There is a great deal of evidence 
pointing to the poor social functioning of people with schizophrenia. Indeed, it is this that makes the illness so very disabling. People with bipolar disorder seem to be more successful at making relationships (although they may have difficulty maintaining them over long periods of time), than those with schizophrenia. They have higher marital rates and higher fertility rates, although studies show that their fertility rates are lower than those in the general population (Lane 1995; Howard 2002). Vogel (1979) found that earlier onset of illness (psychic disturbance before the age of 15) is associated with a worse reproductive outcome and suggested that this is related to the greater personality disturbance seen in those with early-onset disease. It is of note that duration of illness was unrelated to fertility rates.

A qualitative study of people with severe mental illness found that sexual inactivity could be the result of their feelings of anxiety about having to 'come out' about their illness or having to explain their need for psychotropic medication (Wright 2007). In addition, it was found that some patients felt devalued and expected stigmatisation as a result of their diagnosis, leading to intentional celibacy.

Comorbid psychiatric disorders, including generalised anxiety disorder, depression, gender identity disorder, body dysmorphic disorder and alcohol dependency syndrome, may also contribute to sexual dysfunction.

\section{Evaluation}

A sexual history should form part of a thorough initial psychiatric assessment, prior to prescription of medication (Box 2). Change in sexual function should be enquired about on subsequent visits. It may be helpful to use rating scales to monitor any change more accurately (Lingjaerde 1987; Smith 2002). Open discussion of the problem may help to reduce non-adherence to medication later on.

Patients are unlikely to spontaneously mention any sexual problems and direct questioning is required to determine their presence (Knegtering 2004). Inform them that sexual difficulties are common in the general population (Johannes 2000). Let them know that psychiatric medication can cause sexual side-effects just as it can cause other side-effects and that it is therefore important to determine their baseline sexual function. Normalise the experience by reminding them of the usual process of sexual intercourse and the problems that people might encounter if experiencing sexual dysfunction. Remember that lack of sexual activity may be a sign of low libido.
B0X 2 Sexual history
In addition to a thorough psychiatric history and mental state examination, the following information should be gathered

- Current sexual activity

- Who the current partner is and for how long

- Whether there are any relationship difficulties

- Number of previous sexual partners

- Any sexual problems

- Duration of any sexual problems

- The relationship of the sexual problem to time, place or partner

- Any loss of sex drive
- Medical history: any painful conditions such as arthritis, affecting either partner diabetes heart disease, hypertension endocrine disorders such as hypothyroidism, low testosterone levels operations or traumas, especially gynaecological or involving the prostate

- All prescribed medications should be checked for potential sexual side-effects; antihypertensives and diuretics in particular are prone to causing sexual dysfunction

(Based on Tomlinson 1998)
Lack of morning erections and delayed or absent ejaculation are more indicative of an organic than of a psychological problem.

In patients with erectile dysfunction (Box 3), a sex hormone profile may help to determine the cause. Hypogonadism is a reversible cause. A morning blood sample (between 08.00 and $11.00 \mathrm{~h}$ ) should be taken to check testosterone levels. If a low serum testosterone level is found, the test should be repeated along with tests of serum follicle stimulating hormone, luteinizing hormone and prolactin levels (Hackett 2007). If these results are abnormal, a specialist referral should be considered.

\section{B0X 3 Erectile dysfunction}

- Risk factors for erectile dysfunction include obesity, smoking, metabolic syndrome, hypercholesterolaemia and sedentary lifestyle

- Erectile dysfunction is a risk factor for cardiovascular disease

- Erectile dysfunction may be the initial presenting feature of diabetes, hypertension or cardiovascular disease

- Investigations should include a sex hormone profile, fasting glucose and lipids, full blood count and liver function tests

- The phosphodiesterase type-5 (PDE-5) inhibitors sildenafil, tadalafil and vardenafil are licensed for the treatment of erectile dysfunction and prescribed at a maximum of one dose per 24 hours. Usually, only one or two doses are prescribed per week

(Based on Hackett 2007) 
The risk factors for erectile dysfunction are similar to those for cardiovascular disease and include smoking, obesity, metabolic syndrome, hypercholesterolaemia and sedentary lifestyle (Hackett 2007). Erectile dysfunction may be the first presentation of diabetes, hypertension or cardiovascular disease and is itself a cardiovascular risk factor (Hackett 2007; Haffner 2000; Thompson 2005). Therefore, other investigations that should be performed as routine in cases of sexual dysfunction include fasting glucose and lipids, as well as full blood count and liver function tests. If these results are abnormal, a referral to an endocrinologist should be considered.

\section{Managing antipsychotic-induced sexual dysfunction}

Sexual dysfunction induced by antipsychotics is often dose-related, and therefore a reduction in dose may relieve the symptoms. After reducing the dose, wait $6-8$ weeks to see whether there is any improvement. If there is no improvement within this time, or if the reduction in dose does not provide clinical stability, consider switching to a medication with fewer sexual side-effects. In women, this is likely to be a prolactin-sparing medication such as quetiapine; in men, one with fewer anticholinergic or anti-adrenergic sideeffects, such as aripiprazole. If the underlying cause of sexual dysfunction is not clear, the patient should be referred to their general practitioner for a full physical work-up. A specialist referral may follow, if necessary, depending on the organic cause identified, for example to a diabetologist if poorly controlled diabetes is identified as the cause.

In cases of erectile dysfunction, if the patient cannot tolerate a reduction of dose or a switch, it may be necessary to institute a drug to treat it, such as a phosphodiesterase type-5 (PDE-5) inhibitor. A Cochrane review of the evidence base for the management of antipsychotic-induced sexual dysfunction found a dearth of evidence in this area, other than a small trial which reported that sildenafil was helpful (Berner 2007). Drugs that inhibit PDE-5 cause vasodilation, smoothmuscle relaxation and increased arterial blood flow, leading to penile erection (Corbin 2002). These agents have a $75 \%$ success rate when used in attempting sexual intercourse (Hackett 2007). Of the three licensed for use in erectile dysfunction (sildenafil, tadalafil and vardenafil), tadalafil has the longest half-life (17.5 h v. $4 \mathrm{~h}$ for the other two) and has been shown to be the agent of preference among patients (Eardley 2005; Hackett 2007). The PDE- 5 inhibitors are typically prescribed on a once-weekly basis and all have a maximum of one dose in 24h (British Medical Association 2010). They are absolutely contraindicated in patients receiving nitrates (e.g. isosorbide mononitrate, glyceryl trinitrate), because of the risk of unpredictable, potentially dangerous, falls in blood pressure (Hackett 2007). However, they may be taken in conjunction with antihypertensives with only a small risk of significant orthostatic hypotension.

Psychosexual therapy may be indicated if the sexual dysfunction is attributed to psychological or relational difficulties. A Cochrane review of treatment for erectile dysfunction reported that focused group psychosexual therapy plus sildenafil significantly increased rates of successful intercourse compared with sildenafil alone (Melnik 2007). However, there is no evidence base for this treatment in patients with psychotic disorders and it may not be easy for them to access it.

Many genitourinary medicine (GUM) clinics offer psychosexual therapy on referral by a general practitioner, although the nature of the treatments provided and the referral criteria are likely to vary between clinics. Nevertheless, these clinics may prove a useful first port of call for signposting to local resources such as voluntary agencies that provide psychosexual therapy (usually for a fee).

\section{Hypersexuality and risky sexual behaviour in psychosis}

Hypersexuality in psychosis may result directly from an increased sexual drive or may be due to sexual disinhibition as part of a more generalised behavioural disinhibition. Increased sexual drive is considered to be associated with limbic and temporal lobe pathology, whereas behavioural disinhibition is thought to be a feature of paralimbic and neocortical frontotemporal lobe pathology (Baird 2007). A study of psychiatric in-patients in New York found that Hasidic Jews were more likely to demonstrate hypersexual behaviour than controls (matched for age, gender and diagnosis), leading the authors to suggest that in addition to organic pathology, cultural factors may also play a role in the presentation of this symptom (Needell 2004).

The risks to the patient when such a symptom is present are considerable and include not just sexually transmitted infections (STIs), but unwanted pregnancies and the breakdown of romantic relationships if the symptom has led to infidelity. Unwanted sexual advances towards others may lead to criminal charges or retaliatory assault. 
Bipolar disorder is associated with high rates of sexual promiscuity (Sacks 1990). A large cohort study in Dunedin (New Zealand) found that young adults with a diagnosis of bipolar mania were 2.5 times more likely to have engaged in risky sexual intercourse and 4.4 times more likely to have contracted STIs than controls. Adults with schizophrenia were also at an increased risk, with results showing them to be 2.1 times more likely to have engaged in risky sexual behaviour and to have 2.3 times the risk of an STI (Ramrakha 2000). It is estimated that as many as $2 \%$ of people with schizophrenia in the UK are HIV sero-positive, compared with an estimated $0.13 \%$ of total UK population (Gray 2002; Health Protection Agency 2009).

In the USA, Coverdale $\&$ Aruffo (1989) found that a third of female in-patients with severe mental illness reported having sexual intercourse without using contraception despite not wanting to become pregnant, with a similar proportion having a history of induced abortions. Miller $\mathcal{E}$ Finnerty (1998) reported that the most common reason women with schizophrenia gave for failing to use contraception was that they did not expect to have sex. They also identified that another important obstacle to family planning by women with schizophrenia-spectrum disorders was their relative lack of knowledge about the subject (e.g. the potential benefits of long-acting, reversible contraception) in comparison with non-mentally ill controls.

\section{Implications for assessments}

These findings reinforce the need for taking a careful sexual history. At-risk patients should be screened for STIs. When treating women of childbearing age, the possibility that they are currently pregnant or will have an unplanned pregnancy in the future must be borne in mind when prescribing. Clinicians should have a low threshold for offering pregnancy testing.

\section{Implications for patient care}

Given that people with severe mental illness are at greater risk of having unwanted pregnancies and acquiring STIs, it would seem reasonable that their care should include education about risky sexual behaviour and advice about the use of contraception. Pregnancy testing and screening for STIs should be arranged when indicated. Although psychiatrists can provide all patients with a basic level of psychoeducation on these topics, individuals deemed to be at particularly high risk should be referred to specialist GUM clinics for screening, treatment and further education. For other patients, a referral to a family planning clinic or their general practitioner for contraceptive advice may be more appropriate.

Careful consideration should be given to hospital admission for acutely ill patients presenting with hypersexual and sexually disinhibited behaviour, given the risks outlined above. If there is evidence that a mentally ill patient is engaging in behaviour that is sexually inappropriate or likely to lead to regret on recovery from the acute episode, the individual may meet the criteria for detention under the Mental Health Act 1983 (amended 2007) if they are unwilling to accept informal admission (Department of Health 2008). Psychiatrists must bear in mind their responsibility in protecting patients from the risk they pose to themselves and others in such cases.

\section{Capacity, sexuality and psychosis}

The Mental Capacity Act 2005 imposes a legal duty on anyone working with individuals who lack capacity in some areas to always consider whether they have capacity to make a particular decision; if they do not have capacity to decide, the duty is to consider what is in their best interests and whether their best interests can be met using proportionate measures (Office of Public Sector Information 2005). ${ }^{\dagger}$ This duty is likely to be reflected on by psychiatrists in a variety of situations relating to sexuality and psychosis. Specific scenarios in which a psychiatrist might be concerned about a potential need to document their thinking on issues regarding capacity include patients' decisions to, for example, get married, be tested and treated for an STI and to consent to taking contraceptive medications.

Section 27 of the Mental Capacity Act states that nothing in the Act permits a decision to be made on someone else's behalf in relation to their consenting to marriage or a civil partnership, or consenting to have sexual relations. However, the Act's code of practice (Department for Constitutional Affairs 2007) makes it clear that this does not prevent action being taken to protect a vulnerable person from abuse or exploitation. Therefore, if a psychiatrist believes that a person is making such decisions under the influence of a mental disorder, the Mental Capacity Act does not remove the psychiatrist's responsibility to carefully consider taking action, perhaps under other legislation (such as the Mental Health Act), if appropriate.

\section{Sexually transmitted infections}

Given the documented high risk of STIs among people with psychotic illnesses, it may be in a

\section{This issue has recently been addressed in Advances in Biswas $A B$, Hiremath A (2010) Mental capacity assessment and 'best interests' decision-making in clinica practice: a case illustration. 16 :} 440-7. Ed. 
patient's best interests to be screened for infection, particularly if the individual has a known history of risky sexual behaviour. Also, the differential diagnosis of a patient with psychosis may include organic conditions such as HIV infection.

However, it is not difficult to envisage a scenario in which an acutely ill patient holds grandiose or persecutory delusional beliefs that cause them to refuse to have investigations for STIs. They might, for example, believe that their blood will be tampered with, or that they are immune from problems such as STIs. If their reasoning is based on psychotic thinking, then it is highly likely (but not absolutely certain) that they will lack the capacity to make this decision and, for some, it will be considered to be in their best interests to be tested for STIs. Whether it is proportionate to restrain an unwilling, incapacitous individual for the purposes of taking blood for testing will depend on the likelihood that they have an STI and would come to harm because of it if the investigation is delayed until capacity is regained. This will have to be decided on a case-by-case basis. It is essential that the decision-making process is welldocumented. In cases where there is a high index of suspicion that a patient lacking capacity has an STI associated with potentially significant morbidity if left undiagnosed or untreated (e.g. HIV), it would be advisable to seek a second opinion on capacity and best interests before reaching a final decision.

Treatment of STIs in patients lacking capacity can be given under the Mental Capacity Act if it is deemed to be in the best interests of the patient and proportionate. If the STI is causing the mental disorder, as in HIV-related cognitive impairment, it may be possible to treat it under the Mental Health Act.

\section{Unwanted pregnancy and contraception}

Given that severe mental illness is associated with high-risk sexual behaviour and unwanted pregnancies (Coverdale 1989), it may be considered appropriate to offer some female patients oral contraceptives. For some women, it will simply be a matter of providing the necessary information to enable them to make a fully informed decision. However, there will be others who, despite the best efforts of the treating psychiatrist or general practitioner, continue to lack capacity to consent to this treatment. This raises potentially challenging issues about best interests as, essentially, one is deciding whether somebody else should be able to become pregnant. In the case of women prescribed highly teratogenic agents such as sodium valproate, the issue is relatively more easily resolved, given the high risks to the fetus and the consequent accepted best practice of co-prescribing contraception in women of childbearing age. Otherwise, it is understandable that psychiatrists may feel uncomfortable in making such a complex best interests judgement.

Any decision to prescribe contraceptive agents for incapacitous patients should be preceded by exhaustive, documented attempts to maximise the patient's ability to take part in the decisionmaking process. Collateral information should be obtained to gain an understanding of what choice the woman would most likely have made if she did have capacity, as this will form the basis of deciding her best interests. It may be advisable to hold a formal best-interests meeting with all interested parties, including carers and next of kin, to ensure transparency and optimal decision-making. This meeting should include the patient's general practitioner who, as prescriber, will ultimately bear responsibility for the decision to prescribe contraception and for the capacity assessment.

\section{Conclusions}

Issues of sexuality in psychosis can be dealt with confidently by psychiatrists. Alternative antipsychotic agents and the availability of PDE-5 inhibitors allow us to intervene in cases of sexual dysfunction. Greater recognition of the relatively high incidence of risky sexual behaviour in this vulnerable patient group should prompt us to take more careful sexual histories and investigate as appropriate. We must also recognise that these issues can raise complex capacity decisions, and embrace the fact that psychiatrists are well placed to give expert opinions on them.

\section{References}

Aizenberg D, Zemishlany Z, Dorfman-Etrog P, et al (1995) Sexual dysfunction in male schizophrenic patients. Journal of Clinical Psychiatry 56: 137-41

Aizenburg D, Sigler M, Zemishlany Z, et al (1996) Lithium and male sexual function in affective patients. Clinical Neuropharmacology 19: 515-9.

Baird AD, Wilson SJ, Bladin PF, et al (2007) Neurological control of human sexual behaviour: insights from lesion studies. Journal of Neurology, Neurosurgery and Psychiatry 78: 1042-9.

Beasley CM Jr, Tollefson GD, Tran PV (1997) Safety of olanzapine. Journal of Clinical Psychiatry 58 (suppl 10): 13-7.

Berner MM, Hagen M, Kriston L, et al (2007) Management of sexual dysfunction due to antipsychotic drug therapy. Cochrane Database of Systematic Reviews issue 1: CD003546, doi 10.1002/14651858. CD003546.pub2.

Bobes J, Garc A, Portilla MP, et al (2003) Frequency of sexual dysfunction and other reproductive side-effects in patients with schizophrenia treated with risperidone, olanzapine, quetiapine, or haloperidol: the results of the EIRE study. Journal of Sexual and Marital Therapy 29: $125-47$. 
British Medical Association, Royal Pharmaceutical Society of Great Britain (2010) British National Formulary 59 (March). BMJ Group \& RPS Publishing.

Compton MT, Saldivia A, Berry SA, et al (2000) Recurrent priapism during treatment with clozapine and olanzapine. American Journal of Psychiatry 157: 639

Corbin JD, Francis SH, Webb DJ (2002) Phosphodiesterase type 5 as a pharmacologic target in erectile dysfunction. Urology 60: 4-11.

Coverdale JH, Aruffo JA (1989) Family planning needs of female chronic psychiatric outpatients. American Journal of Psychiatry 146: 1489-91.

Department for Constitutional Affairs (2007) Mental Capacity Act 2005: Code of Practice. TSO (The Stationery Office).

Department of Health (2008) Code of Practice: Mental Health Act 1983. TSO (The Stationery Office).

Eardley I, Mirone V, Montorsi F, et al (2005) An open-label, multicentre, randomized, crossover study comparing sildenafil citrate and tadalafil for treating erectile dysfunction in men naïve to phosphodiesterase 5 inhibitor therapy. British Journal of Urology International 96 : 1323-32.

Ghadirian AM, Chouinard G, Annable L (1982) Sexual dysfunction and plasma prolactin levels in neuroleptic-treated schizophrenic outpatients. Journal of Nervous and Mental Disease 170: 463-7.

Ghadirian A, Annable L, Belanger MC (1992) Lithium, benzodiazepines and sexual function in bipolar patients. American Journal of Psychiatry 149: $801-5$.

Gitlin MJ (1994) Psychotropic medications and their effects on sexual function: diagnosis, biology and treatment approaches. Journal of Clinical Psychiatry 55: 406-13.

Gray R, Brewin E, Noak J, et al (2002) A review of the literature on HIV infection and schizophrenia: implications for research, policy and clinical practice. Journal of Psychiatric and Mental Health Nursing 9: $405-9$.

Hackett G, Dean J, Kell P, et al (2007) British Society for Sexual Medicine Guidelines on the Management of Erectile Dysfunction. BSSM (http://www.bssm.org.uk/downloads/BSSM_ED_Management_ Guidelines_2007.pdf).

Haffner SM (2000) Sex hormones, obesity, fat distribution, type 2 diabetes and insulin resistance: epidemiological and clinical correlation. International Journal of Obesity Related Metabolic Disorders 24 (suppl 2): s56-8.

Health Protection Agency (2009) HIV in the United Kingdom: 2009 Report. HPA (http://www.hpa.org.uk/web/HPAwebFile/HPAweb_C/ 1259151891830)

Howard LM, Kumar CK, Leese M, et al (2002) The general fertility rate in women with psychotic disorders. American Journal of Psychiatry 159: 991-7.

Johannes CB, Araujo AB, Feldman HA, et al (2000) Incidence of erectile dysfunction in men 40 to 69 years old: Iongitudinal results from the Massachusetts male aging study. Journal of Urology 163: 460-3.

Jones PB, Bebbington P, Foerster A, et al (1993) Premorbid social underachievement in schizophrenia. Results from the Camberwell Collaborative Psychosis Study. British Journal of Psychiatry 162: 65-71.

Keefe RS, Mohs RC, Losonczy MF, et al (1989) Premorbid sociosexual functioning and long-term outcome in schizophrenia. American Journal of Psychiatry 146: 206-11.

Keller R, Mongini F (2002) Switch to quetiapine in antipsychotic agentrelated hyperprolactinaemia. Neurological Science 23: 233-5.

Kim KS, Pae CU, Chae JH, et al (2002) Effects of olanzapine on prolactin levels of female patients with schizophrenia treated with risperidone. Journal of Clinical Psychiatry 63: 408-13.

Knegtering R, Castelein S, Bous H, et al (2004) A randomized openlabel study of the impact of quetiapine versus risperidone on sexual functioning. Journal of Clinical Psychopharmacology 24: 56-61.
Kotin J, Wilbert DE, Verburg D, et al (1976) Thioridazine and sexual dysfunction. American Journal of Psychiatry 133: 82-5.

Lambert MV (2001) Seizures, hormones and sexuality. Seizure 10 $319-40$.

Lane A, Byrne M, Mulvany F (1995) Reproductive behaviour in schizophrenia relative to other mental disorders: evidence for increased fertility in men despite decreased marital rate. Acta Psychiatrica Scandinavica 91: 222-8.

Lewis R, Bagnall A, Leitner M, et al (2000) Sertindole for schizophrenia. Cochrane Database of Systematic Reviews issue 2: CD001715, doi 10.1002/14651858.CD001715.pub2.

Lingjaerde 0, Ahlfors UG, Bech P, et al (1987) The UKU side-effect rating scale. A new comprehensive rating scale for psychotropic drugs and a cross-sectional study of side-effects in neuroleptic-treated patients. Acta Psychiatrica Scandinavica Supplementum 334: 1-100.

Melnik T, Soares B, Nasello AG (2007) Psychosocial interventions for erectile dysfunction. Cochrane Database of Systematic Reviews issue 3: CD004825, doi 10.1002/14651858.CD004825.pub2.

Miller LJ, Finnerty M (1998) Family planning knowledge, attitudes and practices in women with schizophrenic spectrum disorders. Journal of Psychosomatic Obstetrics and Gynaecology 19: 210-7.

Mitchell JE, Popkin K (1983) Antipsychotic drug therapy and sexual dysfunction in men. American Journal of Psychiatry 139: 633-7.

Nathan RS, Sachar EJ, Ostrow L, et al (1983) A preliminary study of sexrelated differences in prolactin responses to dopamine blockade and insulin hypoglycemia and in penfluridol plasma levels in schizophrenic patients. Psychopharmacology 80: 46-9.

Needell NJ, Markowitz JC (2004) Hypersexual behaviour in Hasidic Jewish inpatients. Journal of Nervous and Mental Disease 192: 243-6.

Office of Public Sector Information (2005) Mental Capacity Act 2005: Chapter 9. OPSI (http://www.opsi.gov.uk/acts/acts2005/pdf/ ukpga_20050009_en.pdf).

Ramrakha S, Caspi A, Dickson N, et al (2000) Psychiatric disorders and risky sexual behaviour in young adulthood: cross sectional study in birth cohort. BMJ 321: 263-6.

Rao VA, Bishop M, Coppen A (1980) Clinical state, plasma levels of haloperidol and prolactin: a correlation study in chronic schizophrenia. British Journal of Psychiatry 137: 518-21.

Rosen SI, Hanno PM (1992) Clozapine-induced priapism. Journal of Urology 148: 876-7.

Sacks MH, Silberstein C, Weiler P, et al (1990) HIV-related risk factors in acute psychiatric inpatients. Hospital and Community Psychiatry 41: 449-51.

Seftel AD, Saenz de Tejada I, Szetela B, et al (1992) Clozapineassociated priapism: a case report. Journal of Urology 147: 146-8.

Smith S, O'Keane V, Murray R (2002) Sexual dysfunction in patients taking conventional antipsychotic medication. British Journal of Psychiatry 181: 49-55.

Smith S, Wheeler MJ, Murray R et al (2003) The effects of antipsychotic-induced hyperprolactinaemia on the hypothalamicpituitary-gonadal axis. Journal of Clinical Psychopharmacology 22: 109-14.

Thompson IM, Tangen CM, Goodman PJ, et al (2005) Erectile dysfunction and subsequent cardiovascular disease. JAMA 294: 2996-3002.

Tomlinson J (1998) Taking a sexual history. BMJ 317: 1573-6.

Vogel HP (1979) Fertility and sibship size in a psychiatric patient population. A comparison with national census data. Acta Psychiatrica Scandinavica 60: 483-503.

Wright ER, Wright DE, Perry BL, et al (2007) Stigma and the sexual isolation of people with serious mental illness. Social Problems 54 78-98.
MCO answers

$1 \mathrm{c} \quad 2$ a $3 \mathrm{~d} \quad 4 \mathrm{~d} 5 \mathrm{e}$ 


\section{MCOs}

Select the single best option for each question stem

1 The poor sexual and reproductive function seen in schizophrenia is:

a a result of dopaminergic dysfunction

b primarily an inherent part of the disease

c secondary to the side-effects of antipsychotic medication

$\mathrm{d}$ associated with an increase in auto-erotic activity

e known to be a result of the high levels of sexual trauma experienced by individuals with psychosis.

2 The following atypical antipsychotic is particularly associated with sexual dysfunction:

a risperidone

b quetiapine c olanzapine

d sertindole

e aripiprazole.

3 PDE-5 inhibitors:

a can be prescribed twice daily in younger males

b elevate blood pressure

c are contraindicated in patients with hypertension

d are contraindicated in patients prescribed nitrates

e reduce blood flow to the penis.

4 People with schizophrenia:

a have higher fertility rates than those with affective psychoses

b have higher rates of sexually transmitted infections than those with affective psychoses

c are likely to readily inform their doctor of sexual dysfunction $\mathrm{d}$ are at increased risk of acquiring HIV

e are likely to use contraception to avoid pregnancy.

5 The Mental Capacity Act 2005:

a can be used to prevent incapacitous people getting married

b can never be used to compel detained patients to have STI testing

c has no bearing on decisions to prescribe contraception for an incapacitous patient detained under the Mental Health Act

$\mathrm{d}$ can be used to prevent incapacitous people having sexual relations

e obliges clinicians making a decision regarding an incapacitous patient to consider whether the action is proportionate and in the patient's best interests. 